
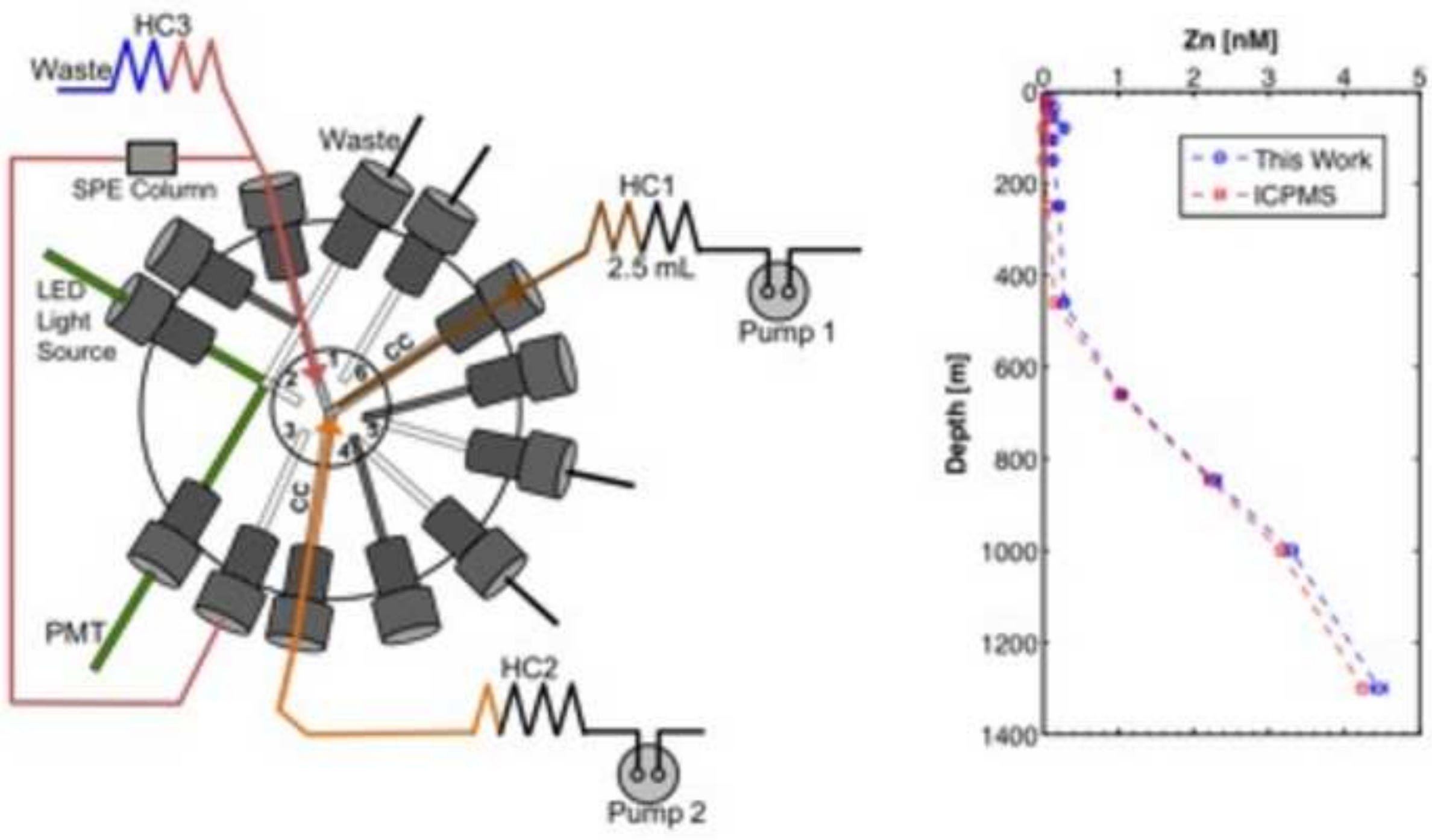


\title{
Determination of trace zinc in seawater by coupling solid phase extraction and fluorescence detection in the Lab-On-Valve format
}

\author{
Maxime M. Grand ${ }^{1,2}$, Petr Chocholouš ${ }^{3}$, Jarda Růžička ${ }^{1}$, Petr Solich $^{3}$ and Christopher I. \\ Measures ${ }^{1}$ \\ ${ }^{1}$ University of Hawaii, Department of Oceanography, Honolulu, HI, USA \\ ${ }^{3}$ Charles University in Prague, Faculty of Pharmacy, Department of Analytical Chemistry, \\ Hradec Kralove, Czech Republic
}

\footnotetext{
${ }^{2}$ Present address: Ocean and Earth Science, National Oceanography Centre Southampton, University of Southampton Waterfront Campus, European Way, Southampton SO14 3ZH, United Kingdom.

Phone: +44(0)2380596475

M.Grand@noc.soton.ac.uk
} 


\section{Abstract}

By virtue of their compactness, long-term stability, minimal reagent consumption and robustness, miniaturized sequential injection instruments are well suited for automation of assays onboard research ships. However, in order to reach the sensitivity and limit of detection required for open-ocean determinations of trace elements, it is necessary to preconcentrate the analyte prior its derivatization and subsequent detection by fluorescence. In this work, a novel method for the determination of dissolved zinc $(\mathrm{Zn})$ at subnanomolar levels in seawater is described. The proposed method combines, for the first time, automated matrix removal, extraction of the target element, and fluorescence detection within a miniaturized flow manifold, based on the Lab-On-Valve (LOV) concept. The key feature of the microfluidic manipulation of the sample is flow programming, designed to pass sample through a mini-column where the target analyte and other complexable cations are retained, while the seawater matrix is washed out. Next, zinc is eluted and merged with a Zn selective fluorescent probe (FluoZin-3) at the confluence point of the LOV central channel using two high-precision stepper motor driven pumps that are operated in concert. Finally, the thus formed $\mathrm{Zn}$ complex is transported to the LOV flow cell for selective fluorescence measurement. This work describes the characterization and optimization of the method including Solid Phase Extraction using the Toyopearl AF-Chelate-650M resin, and detailed assay protocol controlled by a commercially available software and instrument. The proposed method features a LOD of $0.02 \mathrm{nM}$, high precision (<3\% at 0.1 and $2 \mathrm{nM} Z \mathrm{Zn}$ levels), an assay cycle of 13 min and a reagent consumption of $150 \mu \mathrm{L}$ FluoZin-3 per sample, which makes the method highly suitable for oceanographic shipboard analysis. The accuracy of the method has been validated through the analysis of seawater reference standards and comparison with ICP-MS determinations on seawater samples collected in the upper $1300 \mathrm{~m}$ of the subtropical south Indian Ocean. This work confirms that integration of sample pretreatment with optical detection in the LOV format offers a widely applicable approach to trace analysis of seawater.

Keywords: GEOTRACES, Zn, Lab-On-Valve, FluoZin-3, oceanography, trace analysis 


\section{Introduction}

Zinc $(\mathrm{Zn})$ is an essential cofactor of several metalloenzymes and proteins involved in the metabolism of all marine phytoplankton. The availability of dissolved $\mathrm{Zn}$ in surface waters of the remote ocean may thus impact phytoplankton community composition and also regulate carbon uptake in localized regions [1-5]. For these reasons, oceanographers seek to assemble a global database of $\mathrm{Zn}$ measurements in the oceans in order to decipher the role of $\mathrm{Zn}$ in moderating upper ocean biological processes and quantify its impact on global carbon cycling.

The analysis of dissolved $\mathrm{Zn}$ in the remote ocean is a challenging analytical task. In mid and low latitude surface waters, Zn concentrations are generally less than $0.05 \mathrm{nM}$ and small variations in $\mathrm{Zn}$ concentrations can have profound biological implications. Below the surface, the remineralization of settling organic material produces a gradual increase in $\mathrm{Zn}$ concentrations with depth, with $\mathrm{Zn}$ concentrations reaching up to $9 \mathrm{nM}$ in the bottom waters of the Pacific Ocean [6]. Therefore, the determination of dissolved $\mathrm{Zn}$ in the remote ocean requires highly sensitive, selective and precise methods, with a dynamic range of $\sim 0.02-10 \mathrm{nM} \mathrm{Zn}$. In addition, analytical manifolds must be compact because it is preferable to perform all analyses onboard a ship in order to provide near real time data feedback and check for contamination artifacts.

To date, the majority of shipboard dissolved $\mathrm{Zn}$ analyses are performed using slightly modified versions of the Flow Injection Analysis (FIA) fluorometric method of Nowicki et al. [7]. Although this FIA technique has been used successfully on several shipboard transects and process studies in the open ocean [8-10], this method relies on a poorly selective fluorescent reagent which suffers from significant interferences from $\mathrm{Ca}$, $\mathrm{Mg}$ and $\mathrm{Cd}$ [7]. In addition, FIA manifolds have high reagent consumption rates, necessitate regular calibrations, frequent maintenance and thus require highly trained analysts for routine operation onboard research vessels. For example, over prolonged periods of continuous use (8-10 hours) typical of shipboard determinations, variations in the flow rates of FIA manifolds resulting from the stretching of peristaltic tubing will induce drift and significantly contribute to the combined uncertainty of the measurements [11]. 
Micro Sequential Injection Lab-On-Valve ( $\mu$ SI-LOV) manifolds alleviate the majority of the drawbacks of FIA methods [12-15]. This technique is thus an attractive approach for shipboard analysis of trace metals since LOV manifolds are compact, fully automated, and provide highly precise fluidic manipulations in a miniaturized flow manifold with minimal reagent consumption and waste production. Grand et al. [16] reported the first $\mu$ SI-LOV method for trace $\mathrm{Zn}$ determinations in seawater using a highly selective and sensitive fluorescent probe. However, with a Limit of Detection (LOD) of $\sim 0.3 \mathrm{nM}$ [16], this method is not sensitive enough at the lowest $\mathrm{Zn}$ levels $(<0.1 \mathrm{nM})$ that characterize some regions of the open ocean. To achieve a lower LOD using the chemistry of Grand et al. [16], a Solid Phase Extraction (SPE) LOV protocol must be coupled to the fluorometric detection in order to preconcentrate the analyte from solution.

Several protocols for the preconcentration and detection of trace metal cations in the LOV format have recently been developed. Horskotte et al. [17] described a LOV preconcentration manifold coupled with an external $14 \mathrm{~cm}$ flow cell for spectrophotometric detection of Fe using the 1,10-phenantroline chromogenic reagent. Although this work is valuable for absorbance assays requiring long pathlength flowcells to reach adequate analytical sensitivity, detection within the LOV is preferable for ultra trace analyses in order to minimize dispersion of the eluate prior to detection. An alternative approach is to preconcentrate the analyte within the LOV flowcell and monitor the product directly on the surface of the sorbent, as described in the solid phase spectrophotometric LOV method of Santos et al. [18] for the determination of Cd, Zn and $\mathrm{Cu}$ in freshwater samples. However, monitoring fluorescence emission directly on the surface of a sorbent is a challenging task, especially for ultra trace determinations.

This work couples a SPE protocol with fluorescence detection in a single LOV manifold in order to attain sub-nanomolar sensitivity for Zn using the FluoZin-3 fluorescent reagent described in Grand et al. [16]. This is achieved using a commercially available $\mu$ SI-LOV analyzer, furnished with two milliGAT pumps and a LOV equipped with a merging central channel. The present work describes, for the first time, fluorescence detection via merging of reagent and analyte at the confluence point of the LOV central channel and its coupling with SPE. The accuracy of the resulting method 
has been validated using seawater reference materials and seawater samples collected in the subtropical South Indian Ocean.

\section{Materials and Methods}

\subsection{Reagents}

Trace metal clean protocols were employed throughout this work in order to minimize the possibility of Zn contamination. All solutions were prepared using ultra high purity (UHP) water (resistivity $>18.0 \mathrm{M} \Omega \mathrm{cm}$ ) collected from a Nanopure system (Barnstead International, USA) and were stored in acid-washed plastic bottles. Purified solutions of hydrochloric acid (6 M) and glacial acetic acid (17.4 M) were prepared by single distillation of the analytical grade acids (Fisher Scientific, Certified A.C.S Plus) in a quartz-finger still. High purity ammonium hydroxide $\left(\mathrm{NH}_{4} \mathrm{OH}, \sim 5 \mathrm{M}\right)$ was prepared by vapor phase equilibration, as described in Measures et al. [19]. Whenever practical, all solutions were prepared and handled in a Class-100 laminar flow bench to prevent contamination.

\section{Ammonium acetate buffers and acid solutions}

Ammonium acetate buffers (2M) for sample ( $\mathrm{pH} 9.1)$ and reagent $(\mathrm{pH} 8.3)$ adjustment were prepared by adding appropriate volumes of purified acetic acid and ammonium hydroxide to UHP water. Purified $6 \mathrm{M} \mathrm{HCl}$ was used to prepare all acid solutions, including the $0.1 \mathrm{M} \mathrm{HCl}$ eluent solution and the $0.5 \mathrm{M} \mathrm{HCl}$ manifold wash solution.

FluoZin-3 fluorescent reagent

Stock solutions of FluoZin-3 tetrapotassium salt (F24194, Molecular Probes, USA) were prepared by adding $500 \mu \mathrm{g}$ of FluoZin-3 into $1000 \mu \mathrm{L}$ of ammonium acetate reagent buffer ( $\mathrm{pH} 8.3$ ). This stock solution was then subdivided into $20,50 \mu \mathrm{L}$ aliquots 
and stored in acid-cleaned micro-centrifuge tubes in the dark and at $-20{ }^{\circ} \mathrm{C}$ until use. Under these storage conditions $\left(-20^{\circ} \mathrm{C}\right)$, we observed no noticeable decrease in the sensitivity of the FluoZin-3 stock solutions for up to one year. The working solutions of FluoZin-3 $(5 \mu \mathrm{M})$ were prepared daily by diluting the thawed FluoZin-3 stocks in 2950 $\mu \mathrm{L}$ of ammonium acetate reagent buffer $(\mathrm{pH} 8.3)$.

Prior to use, the working $5 \mu \mathrm{M}$ FluoZin-3 solution was purified offline using a SPE Flow Injection manifold and a peristaltic pump set at a flow rate of $10 \mu \mathrm{L} \mathrm{s}^{-1}$ (Gilson, USA). This offline SPE cleaning manifold consisted of two, $2 \mathrm{~cm}$ long mini-columns, each having an internal volume of $27 \mu \mathrm{L}$ (GlobalFIA Inc, USA). The two mini-columns, which were packed with Nobias-PA1 (Hitachi High Tech, Japan) and Toyopearl AFChelate-650M (Tosoh Biosciences LCC, Japan) respectively, were placed in series using $0.8 \mathrm{~mm}$ Teflon tubing (Optimize Technologies, USA). Prior to injecting the FluoZin-3 reagent, the SPE cleaning apparatus was conditioned with $\sim 10 \mathrm{~mL}$ of the reagent buffer ( $\mathrm{pH}$ 8.3). After collecting the purified FluoZin-3 solution in a trace metal clean bottle for later use, the mini-columns were cleaned with a $\sim 20 \mathrm{~mL}$ of $0.5 \mathrm{M} \mathrm{HCl}$ cleaning solution to remove all chelated metals.

\section{Low zinc seawater}

Filtered surface seawater $(0.2 \mu \mathrm{m}$, Pall AcroPak 300), collected using trace metal clean protocols [20] in the Solomon Sea during the GEOTRACES GP12 cruise, was used to optimize the experimental protocol and, after purification, as a blank matrix to calibrate the system. The seawater was stored in the dark and at ambient laboratory temperature $\left(\sim 22^{\circ} \mathrm{C}\right)$ in an acid-washed LDPE container $(20 \mathrm{~L})$. One-liter aliquots of the seawater were then pre-cleaned to produce low $\mathrm{Zn}(<0.02 \mathrm{nM} \mathrm{Zn})$ seawater by acidifying to $\mathrm{pH}=1.7$ with $6 \mathrm{M} \mathrm{HCl}$, storing for $24 \mathrm{hr}$ and then buffering to $\mathrm{pH} 6.0$ via the addition of $50 \mathrm{~mL}$ of the ammonium acetate sample buffer ( $\mathrm{pH}$ 9.1). This buffered seawater was then purified overnight at $20 \mu \mathrm{L} \mathrm{s}^{-1}$ through the dual mini-column SPE cleaning manifold described above in order to produce the blank matrix $(<0.02 \mathrm{nM} \mathrm{Zn})$ used to calibrate the system. 


\section{Seawater standards and reference seawater samples preparation}

Zinc stock standards were prepared by diluting a $1000 \mathrm{mg} \mathrm{L}^{-1}$ commercial standard (Fluka Chemicals, TraceCERT, Switzerland) in UHP water acidified with 24 $\mathrm{mM} \mathrm{HCl}$. The working $\mathrm{Zn}$ standards $(100 \mathrm{~mL})$ were then prepared daily in HDPE bottles by serial dilutions of the $\mathrm{Zn}$ stock solution in low zinc seawater ( $\mathrm{pH}$ 6.0).

Four community reference seawater samples (SAFe-S, SAFe-D2, GD and GS) were used to validate the method [21]. In addition, filtered $(0.45 \mu \mathrm{m})$, acidified seawater samples ( $\mathrm{pH}=1.7$ ) collected in 2009 in the upper 1,300m of the south Indian subtropical gyre $\left(32.2^{\circ} \mathrm{S}, 111.45^{\circ} \mathrm{E}\right)$ during the US-CLIVAR $\mathrm{CO}_{2}$ Repeat Hydrography $\mathrm{I} 05$ transect $[8,22]$ were analyzed for $\mathrm{Zn}$ using the proposed method. Prior to analysis, $20 \mathrm{~mL}$ aliquots of the stored acidified seawater samples $(\mathrm{pH}=1.7)$ were pipetted into $30 \mathrm{~mL}$ PTFE bottles and then buffered offline to $\mathrm{pH} 6.0$ by adding $1 \mathrm{~mL}$ of ammonium acetate sample buffer (pH 9.1).

\subsection{Experimental $\mu$ SI-LOV manifold}

All experiments were performed using a commercially available miniSIA-2 instrument (GlobalFIA Inc., USA). This system (Figure 1) was equipped with a six port selection valve and two High Flow milliGAT pumps connected to two holding coils ( $\mathrm{HC} 1$ and $\mathrm{HC} 2$ ). A six-port, custom-made, transparent LOV was affixed to the front of the selection valve in a vertical position (FIALab®, USA). The key feature of this LOV relative to traditional LOV manifolds is that it contained a merging central channel connecting with both pumps and the two holding coils ( $\mathrm{HC} 1$ and $\mathrm{HC} 2$, Figure 1). This setup allowed the merging of the acidic eluate and reagent streams at the confluence point of the central channel (Figure 1). PTFE tubing with an internal diameter of $0.8 \mathrm{~mm}$ was used for all manifold tubing including the three holding coils (Optimize Technologies, USA). The intake lines of both milliGAT pumps were placed in a 1 L PMP bottle filled with fresh UHP water every day.

The Solid Phase Extraction (SPE) column, which was external to the LOV, consisted of a cylindrical mini-column (diameter $=2.54 \mathrm{~mm}$, Length $=6 \mathrm{~mm}$, Internal 
volume $=30 \mu \mathrm{L}$ ) packed with Toyopearl AF-Chelate-650M resin (Tosoh Bioscience LLC, Japan). This resin, which is functionalized with iminodiacetic type ligands, was held in place inside the mini-column using porous polyethylene frits (GlobalFIA Inc, USA). The mini-column was connected to LOV port 3 at the inlet and to a tee-piece that connects LOV port 1 and a $0.5 \mathrm{~mL}$ holding coil (HC3) at the outlet (Figure 1). The PTFE tubing that connects the column to the tee-piece and LOV port 1 was kept as short as possible (< $1 \mathrm{~cm}$ ), in order to minimize dispersion of the acidic eluate on its way to the LOV central channel merging point (Figure 1).

The integrated LOV fluorescence flow cell $(0.5 \mu \mathrm{L})$ was furnished with two optical fibers (600 $\mu \mathrm{m}$ core) with PEEK shield and bare terminations (FIALab®, USA). The fibers were positioned at right angles in the LOV in order to excite the fluorescent product $(494 \mathrm{~nm})$ and monitor the emission $(516 \mathrm{~nm})$. The receiving optical fiber was connected to a SMA fiber optic photomultiplier tube (PMT, 200ms integration time) furnished with a $525 \pm 25 \mathrm{~nm}$ bandpass filter (FIALab®, USA). The illuminating optical fiber was connected to a XLamp XP-E2 blue LED (Cree, USA). The blue Cree XLamp LED was housed in a custom made casing equipped with a $470 \pm 30 \mathrm{~nm}$ filter placed in front of the SMA fiber optical connector (FIALab®, USA). The LED light intensity was controlled by a dimmer to vary the LED brightness from 0 to $100 \%$. To maximize analytical sensitivity for open ocean Zn determinations, the LED intensity was set at $100 \%$.

\section{Figure 1 near here please}

In order to reduce the ambient light from the laboratory, the complete system including the PMT and LED light source was placed in an opaque cardboard box. For extended use (> $6 \mathrm{hrs}$ ), a USB powered portable desk fan was placed in the box in order to dissipate the heat created by the working milliGAT pumps and prevent heat induced degassing in the flow manifold. All fluidic manipulations, instrument control, data acquisition and peak height quantification were carried out using the FloZF 5.2 software installed on a 64-bit Dell laptop computer running Windows 8. All data processing and statistical analyses were performed using Matlab R2014a (MathWorks, USA). 


\subsection{Assay Protocol}

A complete operational cycle included five steps (Figure 1 and Table 1). The mini-column and HC3 were first flushed with eluting acid $(0.1 \mathrm{M} \mathrm{HCl})$ to ensure the manifold was trace metal clean. This cleaning step, performed prior to each extraction, was done by aspirating $500 \mu \mathrm{L}$ of eluting acid into $\mathrm{HC} 1$ and dispensing $850 \mu \mathrm{L}$ back to the column and HC3 (Table 1). Dispensing a larger return volume of solution to the column $(850 \mu \mathrm{L})$ relative to that aspirated into $\mathrm{HC} 1(500 \mu \mathrm{L})$ ensured that the column was filled with UHP water $(\mathrm{pH}=5.5)$ during the seawater loading step that followed. During the loading step (Figure 1A), $1000 \mu \mathrm{L}$ of buffered seawater sample ( $\mathrm{pH}=6.0$ ) was delivered to the column. As the seawater passed through the column, $\mathrm{Zn}$ (and other cations) were extracted onto the chelating sorbent while the matrix flowed to waste via HC3. This step was repeated 4-8 times (i.e, $4 \mathrm{~mL}$ or $8 \mathrm{~mL}$ load volume), depending on the expected $\mathrm{Zn}$ concentrations in the seawater samples to be analyzed (Table 1).

The elution step (Figure 1B) consisted of dispensing $300 \mu \mathrm{L}$ of $0.1 \mathrm{M} \mathrm{HCl}$ through the mini-column and HC3 (Table 1). In the ensuing derivatization step (Figure 1C), the eluate stored in HC3 was merged at the confluence point of the LOV with the FluoZin-3 reagent previously stored in HC2 (Figure 1B). The derivatization was achieved by operating both pumps, with Pump1 aspirating the acidic eluate into HC1 and Pump2 simultaneously dispensing the fluorescent reagent towards $\mathrm{HC} 1$ via the confluence point of the LOV central channel (Figure 1C). This step is one of the key features of the proposed method as it permits the complexation of $\mathrm{Zn}$ present in the acidic eluate with the fluorescent reagent at the near neutral $\mathrm{pH}$ conditions necessary for the stability of the fluorescent complex.

During the last step (Figure 1D), the PMT was set to start acquiring data and the merged eluate/reagent zone stored in $\mathrm{HC} 1(570 \mu \mathrm{L})$ was directed to the flow cell by reversing the flow of Pump1. The signal intensity at peak maximum (captured in a window at 18-28 seconds after flow reversal) was used to construct the calibration curve and to compute sample composition using the FloZF software. The baseline fluorescence obtained at $\mathrm{t}=70 \pm 10$ seconds was averaged and subtracted from the signal. 
At the end of each day, the sample, eluting acid and reagent inlet lines were placed in the $0.5 \mathrm{M} \mathrm{HCl}$ cleaning solution and the system was thoroughly flushed with acid. Prior shutdown, the milliGAT pumps were flushed with 5-10 mL of UHP water to prevent accumulation of salts in the internal wetted parts of the pumps.

Table 1 near here please.

\section{Results and Discussion}

The determination of trace $\mathrm{Zn}$ at the sub-nanomolar level using the proposed method relies on the optimization of two separate events: (1) the derivatization of the acidic eluate $(0.1 \mathrm{M} \mathrm{HCl}+\mathrm{Zn})$ with the FluoZin-3 reagent, which runs quantitatively at a near neutral $\mathrm{pH}$ [16]; (2) the SPE protocol including the positioning of the mini-column in the flow system, $\mathrm{pH}$ effects, extraction flow rates and eluting acid volume and strength. Although the derivatization process occurs after the SPE in the protocol sequence described in Table 1, the system must first be optimized in direct injection mode prior to coupling with the SPE protocol. Thus, in the following, the optimization of the merging, derivatization and fluorescence detection are considered first.

\subsection{Derivatization and fluorescence detection optimization}

The present work introduces, for the first time, mixing of two confluence streams in a $\mu$ SI-LOV system. For this purpose, the LOV module was modified to include a merging central port communicating with two pumps through two holding coils (Figure 1). By operating the milliGAT pumps at suitable flow rates and directions, the fluorescent reagent and the acidic eluate are merged at the confluence point of the LOV central channel and the mixture is delivered to HC1. Further mixing is promoted by flow reversal, when the reaction mixture is propelled from $\mathrm{HC} 1$ towards the LOV flow cell for to monitor the fluorescence (Figure 1D). In comparison to the sequencing of sample and reagent zones used in Sequential Injection LOV manifolds [12,14,16], the merging approach employed in this work increases the surface area of contact between the acidic 
eluate and reagent zones. This merging step thus facilitates the neutralization of the excess acid present in the eluate for the formation of the fluorescent complex and also minimizes refractive index effects by producing a relatively homogeneous zone of product. This novel LOV confluence mixing scheme is one of the key features of the proposed method and will be readily applicable to other spectroscopic assays, which require neutralization of an acid zone with a concentrated buffer prior to derivatization and detection.

\subsubsection{Zone mixing and derivatization in direct injection mode}

To maximize analytical sensitivity while minimizing reagent consumption, several factors must be optimized during the merging of eluate and fluorescent reagent at the central port of the LOV. The parameters include: (1) The ratio of the eluate to reagent being delivered to $\mathrm{HCl}$, which is set by varying the flow rates of each pump; (2) the total volume of solution dispensed into $\mathrm{HC} 1\left(\mathrm{~V}_{\text {acid }}+\mathrm{V}_{\text {reagent }}\right)$, with larger volumes promoting dispersion past the confluence point, (3) the volume of reagent consumed, which should be minimized and (4) the volume of acid eluate $(0.1 \mathrm{M} \mathrm{HCl})$ stored in $\mathrm{HC} 3$ prior to performing the zones merging step.

The optimization of the derivatization via merging and flow reversal was performed using $\mathrm{Zn}$ standard solutions prepared in $0.1 \mathrm{M} \mathrm{HCl}$ and a $5 \mu \mathrm{M}$ FluoZin-3 reagent solution $(\mathrm{pH} 8.3)$. The $\mathrm{Zn}$ standard solutions were prepared using $0.1 \mathrm{M} \mathrm{HCl}$ in order to replicate the expected composition of the eluate for optimal recovery of $\mathrm{Zn}$ from the iminodiacetic sorbent [8-10]. The optimal mixing protocol was determined by comparing the slopes of the calibration curves obtained after mixing the $0.1 \mathrm{M} \mathrm{HCl} \mathrm{Zn}$ standards $(0-50 \mathrm{nM})$ using different eluate to reagent ratios (7:1-7:4) and eluate volumes (225-450 $\mu \mathrm{L}$ ). In order to alter the eluate:reagent volumetric ratio at the confluence point of the LOV central channel, the flow rate of Pump1 (which aspirates the $0.1 \mathrm{M} \mathrm{HCl} \mathrm{Zn}$ standards into $\mathrm{HC} 1$ ) was kept at $7 \mu \mathrm{L} \mathrm{s}^{-1}$ while the flow rate of Pump2 (which dispenses the FluoZin-3 reagent towards HC1) was varied from 1 to $4 \mu \mathrm{L} \mathrm{s}^{-1}$ (Figure 1C). The volume of eluate aspirated by Pump1 was kept constant at $450 \mu \mathrm{L}$, while the volume of reagent dispensed by Pump2 was varied from 60-240 $\mu \mathrm{L}$ depending on the eluate:reagent 
ratio being investigated. For example, to obtain an eluate:reagent ratio of 7:2 at the confluence point of the central LOV channel, Pump1 aspirates $450 \mu \mathrm{L}$ of eluting acid at 7 $\mu \mathrm{L} \mathrm{s}^{-1}$ while Pump2 dispenses $120 \mu \mathrm{L}$ of reagent at $2 \mu \mathrm{L} \mathrm{s}^{-1}$. To obtain an eluate:reagent ratio of 7:3, Pump2 is set to deliver $180 \mu \mathrm{L}$ of reagent at $3 \mu \mathrm{L} \mathrm{s}^{-1}$, while the flow settings of Pump1 are unchanged. To investigate the effect of eluate volume on sensitivity, the same experiments were then repeated using an eluate volume of $225 \mu \mathrm{L}$ instead of the $450 \mu \mathrm{L}$ used previously.

The optimal conditions were selected based on the slope of the calibration curves as well as suitable peak profiles, with broad Gaussian shaped peaks indicative of homogeneous mixing favored over sharp, asymmetric peak profiles. Figure 2A shows that maximum sensitivity was achieved using an eluate to reagent ratio of 7:2 and an eluate volume of $450 \mu \mathrm{L}$. Increasing the eluate to reagent ratio reduces the procedural blank in a quasi-linear fashion (Figure 2B), due to $\mathrm{pH}$ effects and the non-specific fluorescence of the FluoZin-3 reagent, which is proportional to the concentration of FluoZin-3 in the reacting mixture [16]. The optimal eluate to reagent ratio (7:2) and eluate volume $(450 \mu \mathrm{L})$ also yielded a suitable peak shape and was thus selected for the remaining of the optimization work (Figure 3). The smooth nature of the analytical signal is a clear indication that the merging of the reagent and eluate solutions via the central port and ensuing flow reversal have produced a relatively well-mixed zone of product.

\section{Figure 2 and Figure 3, near here please.}

When programming the flow rates, some additional precautions had to be taken. Thus, Pump 1, which aspirates the eluate, must keep aspirating for several seconds after Pump 2 has finished dispensing the fluorescent reagent, in order to prevent dispensing reagent into $\mathrm{HC} 3$, where the plug of eluate is stored following elution of the mini-column (Figure 1 and Table 1). In addition, it was found that the analytical signal was more reproducible when Pump1, which aspirates the eluted zone of analyte, is started prior to Pump2, which dispenses the reagent, and not the other way around (data not shown).

\subsubsection{Fluorescence detection: linear range and primary light intensity}


After selection of the optimal flow rates and volumes of the derivatization step, the fluorescence quantification range was investigated. This was achieved by analyzing $0.1 \mathrm{M} \mathrm{HCl}$ solutions spiked with increasing concentrations of $\mathrm{Zn}(0-400 \mathrm{nM})$ and monitoring the response at two different excitation light intensities (50 and 100\%). The linearity of the resulting calibration curves was assessed by comparing the least squares linear fits of the produced data (i.e., Zn vs peak height) with a general linear model and determining the goodness of fit between the linear model and the data using ANOVA. At $100 \%$ light intensity (Figure 4), the calibration curve was linear up to $25 \mathrm{nM} \mathrm{Zn}$ and showed significant deviations from linearity above this concentration (ANOVA, $\mathrm{F}_{1,11}=2.0$, $P=0.20)$. When the intensity of excitation was reduced twofold (50\%, Figure 4$)$, the linear quantification range was increased to $100 \mathrm{nM}$, while the analytical sensitivity was reduced by a factor of 2.2. Therefore, a simple linear regression should only be used at $\mathrm{Zn}$ concentrations in the reacting mixture below 25 and $100 \mathrm{nM}$ with the LED light set at 100 and 50\% intensity, respectively. Since the large deviations from linearity did not occur at the same concentration of $\mathrm{Zn}$ at 50 and 100\% light intensity (Figure 4), the asymptotic nature of the calibration curves at high $\mathrm{Zn}$ concentrations was most likely due to saturation of the PMT detector rather than a limiting concentration of FluoZin-3 in the reacting mixture.

Figure 4, near here please.

\subsection{Optimization of the SPE protocol}

\subsubsection{SPE mini-column position in flow system, elution profile and column geometry}

To keep the manifold as simple as possible while minimizing dispersion of analyte prior to mixing with the reagent, several column configurations were investigated. The simplest arrangement involved placing the mini-column between $\mathrm{HC} 1$ and the 
central port of the LOV. In this configuration, the SPE step consisted of aspirating buffered sample through the mini-column using Pump1 and then dispensing it back through the mini-column towards the waste line. The elution step was then performed by aspirating the acid through the column using Pump 1. Finally, the eluate and the FluoZin3 reagent were delivered into $\mathrm{HC} 2$ by aspirating the reagent and dispensing the zone of eluate at the confluence point with the LOV in position 6 (Figure 1). Although this flow protocol and column placement could theoretically yield higher sensitivity since the sample and eluting acid go twice through the column (i.e., one way during the aspiration and then the other when dispensed out), the results were not reproducible. This lack of reproducibility was most likely related to cavitation when the sample and eluting acid are aspirated through the column, a process that creates a high pressure drop across the column and facilitates the formation of bubbles in the flow system. This problem was encountered even when using a mini-column with a larger diameter $(\Phi=0.25 \mathrm{~cm})$, which was tested in an attempt to minimize pressure drop issues across the column. The alternative approach, which was adopted in the present protocol, is based on operating the flow through the column in the same direction during preconcentration and elution (i.e., only pushing fluid through the column and never aspirating from it).

After selecting the position of the mini-column into the manifold, the elution profile of the analyte was investigated. The optimal conditions were selected by preconcentrating $2 \mathrm{~mL}$ of a buffered seawater sample spiked with $8 \mathrm{nM} \mathrm{Zn}$ and varying the volume of eluting acid passed through the column from 100 to $450 \mu \mathrm{L}$ prior to the merging step described above. In all experiments, the volumetric ratio of eluting acid aspirated into $\mathrm{HC} 1$ and then dispensed to the mini-column was kept at 1.3. Figure 5 shows that dispensing $300 \mu \mathrm{L}$ of eluting acid towards HC3 during the mini-column elution phase yielded the maximum signal and highest precision relative to smaller and larger volumes. Thus, the optimized mini-column elution step consists of aspirating 400 $\mu \mathrm{L}$ of $0.1 \mathrm{M} \mathrm{HCl}$ into $\mathrm{HC} 1$ followed by delivery of $300 \mu \mathrm{L}$ to the mini-column and $\mathrm{HC} 3$, where the eluate is stored prior to the merging and derivatization steps.

Figure 5 near here, please 
In an attempt to increase the preconcentration factor and analytical sensitivity, we have experimented with a longer SPE column $(\mathrm{L}=20 \mathrm{~mm}$, i.d.=1.6 mm, internal volume $=$ $27 \mu \mathrm{L})$. The impact of column shape on analytical sensitivity was investigated by comparing the slopes of the calibration curves obtained after pre-concentrating $2 \mathrm{~mL}$ of buffered seawater standards. There was no significant difference in the slopes of the calibration curves obtained using the long $(\mathrm{L}=20 \mathrm{~mm})$ and shorter, $(\mathrm{L}=6 \mathrm{~mm}$, internal volume $=30 \mu \mathrm{L}$ ) wide bore mini-column described in the present work (ANCOVA, $\left.\mathrm{F}_{1,16}=1.75, P=0.20\right)$. To minimize backpressure in the system, the wide bore mini-column was thus adopted in all subsequent experiments.

\subsubsection{Sample loading $\mathrm{pH}$ and loading flow rate optimization}

The effect of $\mathrm{pH}$ on $\mathrm{Zn}$ chelating efficiency onto the Toyopearl AF-Chelate-650M sorbent was investigated by measuring 3-point calibration curves $(\mathrm{Zn}=0-8 \mathrm{nM})$ using five different sets of standards with a $\mathrm{pH}$ ranging from 1.9 to 8.2. In these experiments, the sample volume loaded onto the mini-column was $2 \mathrm{~mL}$. Figure 6 shows that analytical sensitivity plateaus when the sample $\mathrm{pH}$ reaches 5.5 , suggesting that sample $\mathrm{pH}$ must be greater than 5.5 for optimal recovery of $\mathrm{Zn}$ onto the Toyopearl AF-Chelate-650M sorbent. Therefore, prior to analysis, all seawater samples were buffered offline to $\mathrm{pH} 6.0$.

\section{Figure 6 near here please}

An efficient analytical throughput is an important feature for shipboard analysis, in order to keep up with the number of samples $(n=24-48)$ that are generally collected every day. Rapid feedback also allows prompt identification of possibly contaminated samples and permits alteration of the sampling scheme when unexpected features are observed. Since the loading of buffered seawater constitutes the longest step in the analytical sequence (Table 1), the loading flow rate has been optimized in order to increase the analytical throughput, while keeping backpressure in the system under the recommended limit in order to maintain safe operation of the milliGAT pumps. 
The effect of the loading flow rate on analytical sensitivity was estimated by comparing the slopes of three calibration curves obtaining after analyzing a suite of seawater samples spiked with $\mathrm{Zn}(0-8 \mathrm{nM})$ at different loading flow rates $(10,20$ and 40 $\left.\mu \mathrm{L} \mathrm{s}^{-1}\right)$. There was no significant difference in analytical sensitivity when the flow rate was increased from 10 to $20 \mu \mathrm{L} \mathrm{s}^{-1}$ (ANCOVA, $\mathrm{F}_{1,12}=0.52, P=0.48$ ). When the loading flow rate was increased to $40 \mu \mathrm{L} \mathrm{s}^{-1}$, a significant drop in sensitivity was observed (ANCOVA, $\mathrm{F}_{1,8}=6.3, P=0.04$ ) relative to that observed at $20 \mu \mathrm{L} \mathrm{s}^{-1}$. However, the drop in sensitivity was only about $13 \%$, which is marginal relative to the increase in analytical throughput obtained at a higher flow rates. We thus selected a loading flow rate of $40 \mu \mathrm{L}$ $\mathrm{S}^{-1}$.

\subsubsection{Eluent strength}

The eluting acid must be sufficiently concentrated to quantitatively desorb $\mathrm{Zn}$ from the Toyopearl AF-Chelate-650M sorbent, but also weak enough so that the acid can be neutralized with the concentrated buffer present in the fluorescent reagent in order to reach the near neutral $\mathrm{pH}$ conditions necessary for the formation of the fluorescent complex. Significantly higher sensitivity was achieved with $0.08 \mathrm{M} \mathrm{HCl}$ relative to $0.1 \mathrm{M}$ $\mathrm{HCl}$ (ANCOVA, $\mathrm{F}_{1,8}=67.8, P<0.01$ ), suggesting that the reaction $\mathrm{pH}$ following mixing of the FluoZin-3 reagent with $0.1 \mathrm{M} \mathrm{HCl}$ probably lies at the lower end $(\sim \mathrm{pH} 6.5)$ of the optimal reaction $\mathrm{pH}$ range [16]. Although the use of a $0.08 \mathrm{M}$ eluting acid increases analytical sensitivity by up to $25 \%$, a $0.1 \mathrm{M} \mathrm{HCl}$ eluting acid was selected since it provided adequate analytical sensitivity without any noticeable carryover when low $\mathrm{Zn}$ seawater samples $(\sim 0.02 \mathrm{nM})$ were analyzed immediately following a high $\mathrm{Zn}$ standard solution (8nM, data not shown).

\subsubsection{Interferences}

In their screening of the selectivity of the FluoZin-3 reagent, Grand et al. [16] suggested that the presence of $\mathrm{Cd}$ in seawater samples may produce a significant positive interference on the $\mathrm{Zn}$ signal. In the present study, the $\mathrm{Cd}$ interference was revisited by 
comparing the signal intensity obtained after loading $3 \mathrm{~mL}$ of a low $\mathrm{Zn}$ seawater sample with that spiked with $1 \mathrm{nM} \mathrm{Zn,} 1 \mathrm{nM} \mathrm{Cd}$ and $1 \mathrm{nM} \mathrm{Zn} \& \mathrm{Cd}$ combined. These experimental conditions constituted a worst-case scenario, considering that the highest $\mathrm{Cd}$ concentrations observed in the open ocean are on the order of $1 \mathrm{nM}$ (Bruland and Lohan, 2003). No significant differences in the mean analytical signal of a low Zn seawater sample and that spiked with $1 \mathrm{nM} \mathrm{Cd}$ were observed (two sample t-test, $P=0.18, \mathrm{df}=7$ ). The mean signals of the $1 \mathrm{nM} \mathrm{Zn}$ standard and that of a $1 \mathrm{nM}$ and $1 \mathrm{nM} \mathrm{Cd}$ mixed seawater standard were also not significantly different (two sample t-test, $P=0.35, \mathrm{df}=4$ ). Therefore, when analyzing seawater samples collected in the open ocean (up to $1 \mathrm{nM} \mathrm{Cd}$ ) using the proposed method, the presence of $\mathrm{Cd}$ in the samples can be safely ignored.

\subsubsection{Blank quantification}

Although possible blank contributions were minimized through purification of all solutions including the FluoZin-3 reagent prior to use, it is still necessary to estimate the magnitude of the procedural blank and correct the data produced. In this work, the procedural blank is estimated each day by double spiking a low Zn seawater sample with sample buffer and analyzing the resulting solution as others. The resulting blank signal $(\sim 0.7 \mathrm{nM})$ is then subtracted from all the data produced. Note that the majority of the blank signal is not specific to $\mathrm{Zn}$ and stems from the auto-fluorescence of the FluoZin-3 reagent [16]. Although the blank signal is large, its magnitude will remain relatively constant over time thanks to the precise fluidic handling provided by the milliGAT pumps and reproducible fluidic manipulations in the LOV. Thus, in spite of the unavoidable non-specific FluoZin-3 blank, subnanomolar accuracy can be readily achieved.

\subsection{Analytical Performance}

The principal analytical features of the optimized system, including the linear calibration range, LOD, precision and analytical throughput are listed in Table 2. For 
open ocean sample determinations, the system was operated with two different sample preconcentration volumes depending on the expected $\mathrm{Zn}$ concentrations in the seawater samples (Table 2). At $\mathrm{Zn}$ concentrations greater than $1 \mathrm{nM}$, such as found throughout the Southern Ocean and in deep waters $(>700 \mathrm{~m})$ of the temperate and tropical oceans, loading $4 \mathrm{~mL}$ of buffered seawater onto the mini-column was sufficient to obtain adequate analytical sensitivity. For near surface seawater samples with $\mathrm{Zn}$ concentrations less than $1 \mathrm{nM}$, the preconcentration volume was increased to $8 \mathrm{~mL}$ in order to enhance analytical sensitivity.

\section{Table 2 near here, please}

\subsubsection{Linear calibration range, LOD and analytical precision}

Figure 7 displays two typical calibration curves obtained following preconcentration of $4 \mathrm{~mL}$ and $8 \mathrm{~mL}$ of buffered seawater $(\mathrm{pH}=6.0)$ onto the mini-column. Using the optimized experimental protocol with both 4 and $8 \mathrm{~mL}$ preconcentration volumes, the calibration range is linear up to $4 \mathrm{nM} \mathrm{Zn}$. Above this concentration, the $\mathrm{Zn}$ vs fluorescence response starts to plateau and it is recommended to decrease the intensity of excitation light source (Figure 8) or to reduce the loading volume in order to extend the dynamic linear range. The latter is easily implemented by altering the number of times the sample loading step is repeated in the protocol sequence (Table 1).

\section{Figure 7 near here, please}

The LOD, defined as three times the standard deviation of the peak heights $(n=3)$ of low zinc seawater injections divided by the slope of the calibration curve, was $0.02 \mathrm{nM}$ $\mathrm{Zn}$ when operating the system with $8 \mathrm{~mL}$ sample load volume and $0.07 \mathrm{nM}$ with $4 \mathrm{~mL}$ sample load volume (Table 2). The analytical reproducibility, defined as the relative standard deviation of replicate analyses of samples containing 0.1 and $2 \mathrm{nM} \mathrm{Zn} \mathrm{(n=3),} \mathrm{is}$ generally less than $3 \%$ (Table 2). 


\subsubsection{Long-term stability of the method}

The stability of the proposed method was established through repeated analyses $(\mathrm{n}=67)$ of a buffered seawater sample $(\mathrm{Zn}=1 \mathrm{nM})$ over a $10.5 \mathrm{hr}$ period using a $4 \mathrm{~mL}$ preconcentration volume. Figure 9 shows that the variability of the signal relative to the first injection never exceeded $\pm 10 \%$ over the course of the stability experiment.

Interestingly, the variations in the analytical signal over time do not appear to be entirely random but appear to follow a sinusoidal trend, which may be related to fluctuations of the laboratory ambient temperature throughout the course of the day (Figure 9). Over the $10.5 \mathrm{hr}$ period, the relative standard deviation of the 67 injections of the $1 \mathrm{nM}$ seawater sample was $4.2 \%$. Thus the proposed method can be used for extended periods in a shipboard or laboratory environment, without frequent recalibration to account for drift.

\section{Figure 9 near here, please}

\subsection{Accuracy assessment and method validation}

The accuracy of the proposed method was validated using community reference standards, which are routinely used in the field of oceanography [21]. In addition, the method was also validated through the analysis of a set of samples collected in the upper $1300 \mathrm{~m}$ of the South Indian Ocean and comparison with data produced via independent ICP-MS determinations at Florida State University using the method of Milne et al. [23].

Excellent agreement between the proposed $\mu$ SI-LOV method and the consensus values was obtained for reference samples GD-62 and SAFe-D2 (Table 3). The Zn concentrations obtained for the reference samples with a consensus value less than 0.1 nM Zn may be slightly overestimated (Table 3). However, considering that the mean values for the reference samples lay within $\pm 1 \sigma$ of the mean consensus values (Table 3 ), it is clear that the present method is capable of providing accurate data over the range of $\mathrm{Zn}$ concentrations that characterize the remote open ocean $(\sim 0.02-9 \mathrm{nM})$. 
Figure 10 compares the $\mathrm{Zn}$ profiles obtained in the South Indian Ocean using the proposed method and through ICP-MS determinations. The Zn profile obtained using the present $\mu$ SI-LOV method was analyzed on two separate days, using a preconcentration volume of $8 \mathrm{~mL}$ in the upper $460 \mathrm{~m}(0.03$ to $0.28 \mathrm{nM})$ and $4 \mathrm{~mL}$ volume below that depth (1.02-4.46 nM) with each sample analyzed in duplicate. A model II linear regression between the ICP-MS and $\mu$ SI-LOV data yields a slope of $1.01 \pm 0.01$ and an intercept of $0.11 \pm 0.02$ with a $\mathrm{R}^{2}$ of $0.998(n=12)$. Even though the $\mu$ SI-LOV data may be slightly overestimated relative to that produced via ICP-MS, the present $\mu$ SI-LOV method produced an oceanographically consistent profile at some of the lowest $\mathrm{Zn}$ concentrations observed in the open ocean. This result further demonstrates the suitability and accuracy of the present method for shipboard open ocean determinations.

Figure 10 near here, please

\section{Conclusions}

This work describes, for the first time, a $\mu$ SI-LOV protocol coupling analyte preconcentration and fluorescence detection in a single LOV manifold, which is applied to the determination of trace $\mathrm{Zn}$ levels in seawater. The long-term stability of the proposed method, dynamic quantification range along with its automated operation, minimal set-up time and maintenance requirements make it a very attractive method for high-throughput shipboard determinations of dissolved $\mathrm{Zn}$ in virtually all areas of the open ocean.

In addition, the present method provides a relatively universal $\mu$ SI-LOV protocol for the preconcentration and spectroscopic determination of transition metal cations in seawater, which could be readily applied to other analytes of interest in oceanography (e.g., $\mathrm{Al}, \mathrm{Fe}, \mathrm{Mn}$ ). For example, the proposed $\mu \mathrm{SI}-\mathrm{LOV}$ manifold and fluidic protocol can be easily converted to absorbance assays simply by repositioning the optical fibers 
around the integrated LOV flow cell, changing the light source and detector and following the steps described in this work to optimize and validate the assay. The manifold could also be improved by incorporating an extra port into the LOV, which could be used to aspirate sample buffer and then perform online buffering of the acidified seawater samples at the confluence point of the LOV. This modification would produce a commercially available miniaturized shipboard analyzer likely to surpass traditional FIA systems, which, in spite of the many developments in flow methods over the past two decades, remain the state of the art in chemical oceanography.

\section{Acknowledgements}

This work was funded by NSF-OCE 1235101 to CIM and by the Czech Ministry of Education, Youth and Sports project VES13 Kontakt II LH13023 to PC and PS. The ICP-MS analyses reported here were performed at the National High Magnetic Field Laboratory, which is supported by the National Science Foundation Cooperative Agreement No DMR-1157490 and the State of Florida. We would like to thank Dr. Pete Morton (FSU) for obtaining and sharing the ICP-MS data presented in this work and Dr. Mariko Hatta (UH) for her help, thoughtful comments and laboratory support. Thanks are also due to the captain and crew of the NO L'Atalante and to Dr. Jérôme Aucan, Dr. François Lacan and Dr. Catherine Jeandel for inviting MG on the GEOTRACES GP12 cruise, during which the trace metal clean seawater used throughout this work was collected. This manuscript benefited from the constructive comments of two anonymous reviewers and is SOEST contribution \#xxxx. 


\section{References}

[1] F.M.M. Morel, J.R. Reinfelder, S.B. Roberts, C.P. Chamberlain, J.G. Lee, D. Yee, Zinc and carbon co-limitation of marine phytoplankton, Nature. 369 (1994) 740742. doi:10.1038/369740a0.

[2] W.G. SUNDA, S.A. HUNTSMAN, Cobalt and zinc interreplacement in marine phytoplankton: Biological and geochemical implications, Limnol. Oceanogr. 40 (1995) 1404-1417. doi:10.4319/lo.1995.40.8.1404.

[3] Y. Shaked, Y. Xu, K. Leblanc, F.M.M. Morel, Zinc availability and alkaline phosphatase activity in Emiliania huxleyi: Implications for $\mathrm{Zn}-\mathrm{P}$ co-limitation in the ocean, Limnol. Oceanogr. 51 (2006) 299-309. doi:10.4319/lo.2006.51.1.0299.

[4] R.W. Jakuba, J.W. Moffett, S.T. Dyhrman, Evidence for the linked biogeochemical cycling of zinc, cobalt, and phosphorus in the western North Atlantic Ocean, Global Biogeochem. Cycles. 22 (2008) n/a-n/a. doi:10.1029/2007GB003119.

[5] W.G. Sunda, Feedback Interactions between Trace Metal Nutrients and Phytoplankton in the Ocean., Front. Microbiol. 3 (2012) 204. doi:10.3389/fmicb.2012.00204.

[6] K.W.W. Bruland, M.C.C. Lohan, Controls of Trace Metals in Seawater, in: K.K. Elderfield, H, Holland, D, Turekian (Ed.), Treatise on Geochemistry, Elsevier, Elsevier, 2003: pp. 23-47. doi:10.1016/B0-08-043751-6/06105-3.

[7] J.L. Nowicki, K.S. Johnson, K.H. Coale, V.A. Elrod, S.H. Lieberman, Determination of Zinc in Seawater Using Flow Injection Analysis with Fluorometric Detection, Anal. Chem. 66 (1994) 2732-2738. doi:10.1021/ac00089a021.

[8] K.J. Gosnell, W.M. Landing, A. Milne, Fluorometric detection of total dissolved zinc in the southern Indian Ocean, Mar. Chem. 132-133 (2012) 68-76. doi:10.1016/j.marchem.2012.01.004.

[9] N.J. Wyatt, A. Milne, E.M.S. Woodward, A.P. Rees, T.J. Browning, H.A. Bouman, et al., Biogeochemical cycling of dissolved zinc along the GEOTRACES South Atlantic transect GA10 at 40 $\mathrm{S}$, Global Biogeochem. Cycles. 28 (2014) 44-56. doi:10.1002/2013GB004637.

[10] D.J. Janssen, J.T. Cullen, Decoupling of zinc and silicic acid in the subarctic northeast Pacific interior, Mar. Chem. 177 (2015) 124-133. doi:10.1016/j.marchem.2015.03.014.

[11] G.H. Floor, R. Clough, M.C. Lohan, S.J. Ussher, P.J. Worsfold, C.R. Quétel, Combined uncertainty estimation for the determination of the dissolved iron amount content in seawater using flow injection with chemiluminescence detection, Limnol. Oceanogr. Methods. 13 (2015) 673-686. doi:10.1002/lom3.10057.

[12] J. Ruzicka, Lab-on-valve: universal microflow analyzer based on sequential and bead injection, Analyst. 125 (2000) 1053-1060. doi:10.1039/b001125h.

[13] J. Ruzicka, Flow Injection Analysis: Tutorial \& News on Flow Based micro Analytical Techniques, (2016). http://www.flowinjectiontutorial.com (accessed January 15, 2016).

[14] R.B.R. Mesquita, A.O.S.S. Rangel, A review on sequential injection methods for 
water analysis., Anal. Chim. Acta. 648 (2009) 7-22.

doi:10.1016/j.aca.2009.06.030.

[15] H.M. Oliveira, M.M. Grand, J. Ruzicka, C.I. Measures, Towards chemiluminescence detection in micro-sequential injection lab-on-valve format: a proof of concept based on the reaction between Fe(II) and luminol in seawater, Talanta. 133 (2015) 107-111. doi:10.1016/j.talanta.2014.06.076.

[16] M.M. Grand, H.M. Oliveira, J. Ruzicka, C.I. Measures, Determination of dissolved zinc in seawater using micro-Sequential Injection lab-on-valve with fluorescence detection., Analyst. 136 (2011) 2747-55. doi:10.1039/c1an15033b.

[17] B. Horstkotte, P. Chocholouš, P. Solich, Large volume preconcentration and determination of nanomolar concentrations of iron in seawater using a renewable cellulose 8-hydroquinoline sorbent microcolumn and universal approach of postcolumn eluate utilization in a Lab-on-Valve system., Talanta. 150 (2016) 213-23. doi:10.1016/j.talanta.2015.12.044.

[18] I.C. Santos, R.B.R. Mesquita, A.O.S.S. Rangel, Micro solid phase spectrophotometry in a sequential injection lab-on-valve platform for cadmium, zinc, and copper determination in freshwaters., Anal. Chim. Acta. 891 (2015) 1718. doi:10.1016/j.aca.2015.08.021.

[19] C.I. Measures, J. Yuan, J.A. Resing, Determination of iron in seawater by flow injection analysis using in-line preconcentration and spectrophotometric detection, Mar. Chem. 50 (1995) 1-10. doi:10.1016/0304-4203(95)00022-J.

[20] C.I. Measures, W.M. Landing, M.T. Brown, C.S. Buck, A commercially available rosette system for trace metal clean sampling, Limnol. Oceanogr. Methods. 6 (2008) 384-394. doi:10.4319/lom.2008.6.384.

[21] K.W. Bruland, GEOTRACES Standard and Reference Materials, (2016). $\mathrm{http}: / / \mathrm{www}$.geotraces.org/science/intercalibration/322-standards-and-referencematerials (accessed January 15, 2015).

[22] M.M. Grand, C.I. Measures, M. Hatta, P.L. Morton, P. Barrett, A. Milne, et al., The impact of circulation and dust deposition in controlling the distributions of dissolved $\mathrm{Fe}$ and $\mathrm{Al}$ in the south Indian subtropical gyre, Mar. Chem. 176 (2015) 110-125. doi:10.1016/j.marchem.2015.08.002.

[23] A. Milne, W. Landing, M. Bizimis, P. Morton, Determination of Mn, Fe, Co, Ni, $\mathrm{Cu}, \mathrm{Zn}, \mathrm{Cd}$ and $\mathrm{Pb}$ in seawater using high resolution magnetic sector inductively coupled mass spectrometry (HR-ICP-MS)., Anal. Chim. Acta. 665 (2010) 200-7. doi:10.1016/j.aca.2010.03.027. 
Table 1. Protocol sequence. The merged rows indicate that the milliGAT pumps are operating in unison. CC: Central Channel.

\begin{tabular}{|c|c|c|c|c|c|c|c|}
\hline Step & \multicolumn{2}{|c|}{ LOV Position } & \multirow{2}{*}{$\begin{array}{l}\text { Pump } \\
\text { Pump 1 }\end{array}$} & \multirow{2}{*}{\begin{tabular}{|l} 
Aspirate/Dispense \\
Aspirate
\end{tabular}} & \multirow{2}{*}{\begin{tabular}{|l} 
Volume $(\boldsymbol{\mu L})$ \\
500
\end{tabular}} & \multirow{2}{*}{\begin{tabular}{|l|}
$\begin{array}{l}\text { Flow rate } \\
(\boldsymbol{\mu L} / \mathbf{s})\end{array}$ \\
50 \\
\end{tabular}} & \multirow{2}{*}{\begin{tabular}{|l|} 
PMT \\
off \\
\end{tabular}} \\
\hline Clean Column & 4 & $0.1 \mathrm{M} \mathrm{HCl}$ & & & & & \\
\hline - & 3 & Column & Pump 1 & Dispense & 850 & 20 & off \\
\hline \multirow{2}{*}{$\begin{array}{l}\text { Load Column with } \\
\text { sample }(n=4-8)\end{array}$} & 5 & Sample & Pump 1 & Aspirate & 1000 & 50 & off \\
\hline & 3 & Column & Pump 1 & Dispense & 1250 & 40 & off \\
\hline \multirow{2}{*}{$\begin{array}{l}\text { Elute Zn from } \\
\text { Column }\end{array}$} & 4 & $0.1 \mathrm{M} \mathrm{HCl}$ & Pump 1 & Aspirate & 400 & 50 & off \\
\hline & 3 & Column & Pump 1 & Dispense & 300 & 10 & off \\
\hline Aspirate Reagent & 6 & FluoZin-3 & Pump 2 & Aspirate & 150 & 10 & off \\
\hline \multirow{2}{*}{$\begin{array}{l}\text { Merge at } \mathrm{CC} \text { and } \\
\text { store in } \mathrm{HC1}\end{array}$} & \multirow{2}{*}{1} & \multirow{2}{*}{$\mathrm{HC} 3$} & Pump 1 & Aspirate & 450 & 7 & \multirow{2}{*}{ off } \\
\hline & & & Pump 2 & Dispense & 120 & 2 & \\
\hline $\begin{array}{l}\text { Fluorescence } \\
\text { Monitoring }\end{array}$ & 2 & Flow Cell & Pump 1 & Dispense & 1200 & 17 & on \\
\hline \multirow{2}{*}{ Flush HC3 } & \multirow{2}{*}{1} & \multirow{2}{*}{$\mathrm{HC} 3$} & Pump 1 & Dispense & 300 & 50 & \multirow{2}{*}{ off } \\
\hline & & & Pump 2 & Dispense & 300 & 50 & \\
\hline \multirow{2}{*}{ Flush Flow Cell } & \multirow{2}{*}{2} & \multirow{2}{*}{ Flow Cell } & Pump 1 & Dispense & 200 & 50 & \multirow{2}{*}{ off } \\
\hline & & & Pump 2 & Dispense & 200 & 50 & \\
\hline
\end{tabular}


Table 2. Figures of merit of the proposed $\mu$ SI-LOV protocol for dissolved $\mathrm{Zn}$ determinations following extraction of 4 and $8 \mathrm{~mL}$ of buffered seawater.

\begin{tabular}{|l|l|l|l|l|}
\hline & $\begin{array}{l}\text { Linear } \\
\text { Calibration } \\
\text { Range } \\
{[\mathrm{nM}]^{*}}\end{array}$ & $\begin{array}{l}\text { LOD } \\
{[\mathrm{nM}]}\end{array}$ & $\begin{array}{l}\text { Repeatability } \\
{[\%]^{* *}}\end{array}$ & $\begin{array}{l}\text { Analytical } \\
\text { Throughput } \\
\text { [min/sample] }\end{array}$ \\
\hline $\begin{array}{l}4 \mathrm{~mL} \\
\text { injection }\end{array}$ & $0-4$ & 0.07 & $\begin{array}{l}0.7-2.5 \% \text { at } 1.7 \mathrm{nM} \mathrm{Zn} \\
0.9-3.1 \% \text { at } 0.07 \mathrm{nM} \mathrm{Zn}\end{array}$ & 9 \\
\hline $\begin{array}{l}8 \mathrm{~mL} \\
\text { injection }\end{array}$ & $0-4$ & 0.02 & $\begin{array}{l}0.8-2.1 \% \text { at } 1.7 \mathrm{nM} \mathrm{Zn} \\
1.1-2.8 \% \text { at } 0.07 \mathrm{nM} \mathrm{Zn}\end{array}$ & \\
\hline
\end{tabular}

*The linear dynamic range can be extended to higher concentrations by reducing the intensity of the excitation light source or lowering the sample volume loaded (see text).

**Analytical repeatability is given as the minimum and maximum relative standard deviations (min-max) of triplicate analyses of reference samples GD $(\sim 1.7 \mathrm{nM})$ and SAFe-S $(\sim 0.07 \mathrm{nM})$. These samples were analyzed each day the system was used during the validation phase of the proposed method. 
Table 3. Analysis of the SAFe and GEOTRACES reference samples and comparison with the consensus values as of May 2013.

(http://www.geotraces.org/index.php/science/intercalibration/322-standards-andreference-materials)

\begin{tabular}{|l|l|l|l|l|}
\hline & GD-62 & GS-144 & SAFe-S 489 & SAFe-D2 366 \\
\hline $\begin{array}{l}\text { This work } \\
\left(\begin{array}{l}\text { mean } \pm 1 \text { S.D }) \\
\text { [nmol kg }\end{array}\right.\end{array}$ & $1.68 \pm 0.08(\mathrm{n}=21)$ & $0.05 \pm 0.02(\mathrm{n}=8)$ & $0.08 \pm 0.02(\mathrm{n}=9)$ & $7.61 \pm 0.25(\mathrm{n}=11)$ \\
\hline $\begin{array}{l}\text { Consensus } \\
\left(\begin{array}{l}\text { mean } \pm \mathbf{1} \text { S.D) } \\
\text { [nmol kg }\end{array}\right.\end{array}$ & $1.71 \pm 0.12$ & $0.041 \pm 0.007$ & $0.069 \pm 0.01$ & $7.43 \pm 0.25$ \\
\hline & & & & \\
\hline
\end{tabular}




\section{Figure Captions}

Figure 1. Schematic of the experimental $\mu$ SI-LOV manifold and four principal steps of the protocol sequence. CC: Central Channel; HC:Holding Coil. Numbers refer to the LOV port numbers.

Figure 2. Optimization of derivatization at the confluence point of the central channel. A: Slope of 3 point calibration curves as a function of the Eluate:Reagent ratio at the confluence point. B: Intercept of the calibration curves as a function of the Eluate:Reagent ratio. See text for details.

Figure 3. Peak profiles of a $25 \mathrm{nM} \mathrm{Zn}$ sample at different Eluate:Reagent (E:R) ratios. The thicker peak (blue) denotes the Eluate:Reagent ratio selected in the present method.

Figure 4. Linear quantification range in direct injection mode at 50 and $100 \%$ intensity. The dashed lines show the least squares fits of the linear part of the quantification range (0-25 $\mathrm{nM}$ at $100 \%$ light intensity, $0-50 \mathrm{nM}$ at 50\% light intensity). Each standard was analyzed in duplicate and the symbols show the mean peak height.

Figure 5. Optimization of the eluting acid volume dispensed into HC3 prior to derivatization. This plot was produced by increasing the volume of eluting acid dispensed into HC3 from 100 to $450 \mu \mathrm{L}$. Error bars shows the standard deviation of duplicate injections. When no error bar is visible, the error is contained within the symbol.

Figure 6. Effect of sample $\mathrm{pH}$ on recovery of $\mathrm{Zn}$ onto the Toyopearl AF-Chelate-650M sorbent. The $\mathrm{y}$-axis is the slope of 3-point calibration curves run at each $\mathrm{pH}$ investigated.

Figure 7. Calibration curves following the preconcentration of 4 and $8 \mathrm{~mL}$ of buffered seawater using the proposed optimized method. Each seawater standard was run in duplicate. The slopes and intercepts of the calibration curves are shown with their respective standard deviations.

Figure 8. Peak profiles resulting from the preconcentration and analysis of seawater standards spiked with increasing $\mathrm{Zn}$ concentrations in triplicate, with the incident light intensity reduced to $75 \%$. The inset shows the calibration curve associated with these injections, with error bars representing the standard deviation of each standard.

Figure 9. Long-term stability experiment. These data were produced by analyzing a 1 nM Zn seawater sample (4 mL load volume) over a $10.5 \mathrm{hr}$ period ( $n=67)$. Each bar represents the percent difference of the peak height at time $=t$ relative to the peak height of the sample obtained at $\mathrm{t}=0$ (see inset equation). Note that 5 injections were excluded from this analysis due to irregular peak shapes, which could not be corrected.

Figure 10. Zn profile from the upper $1300 \mathrm{~m}$ of the South Indian subtropical gyre analyzed using the proposed $\mu$ SI-LOV method and also by ICP-MS. The $\mu$ SI-LOV samples were analyzed in duplicate, error bars show the standard deviation. 

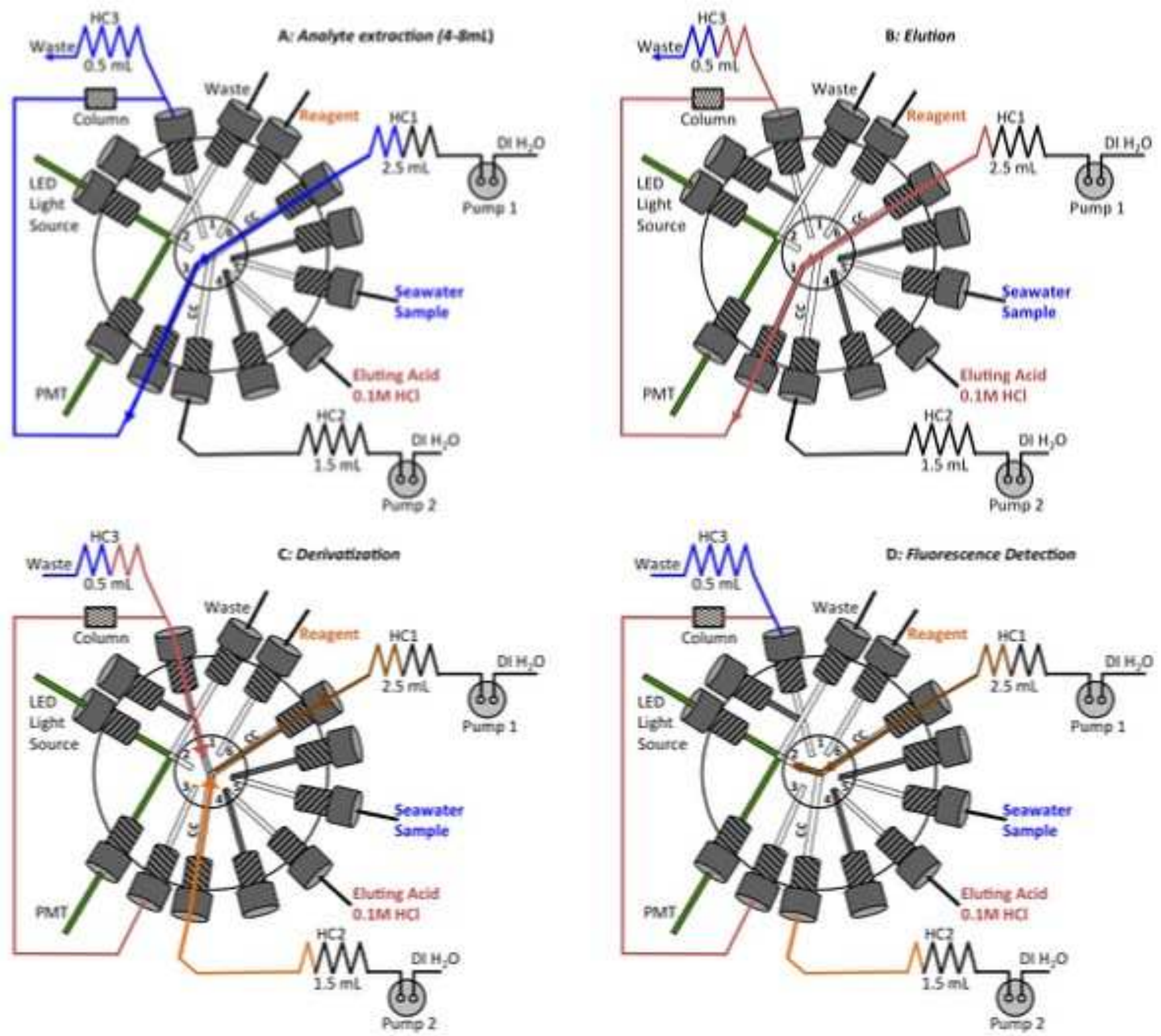

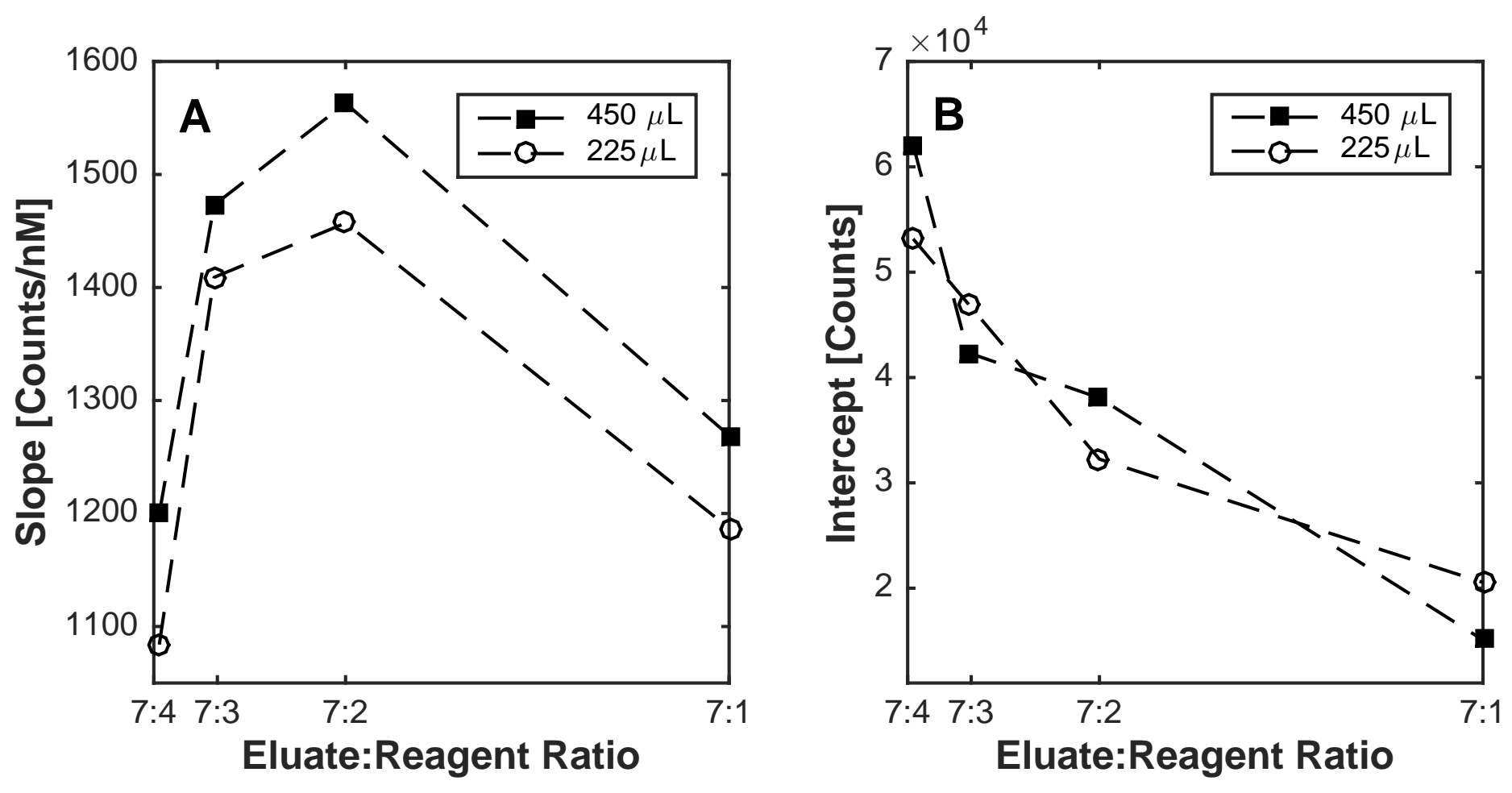


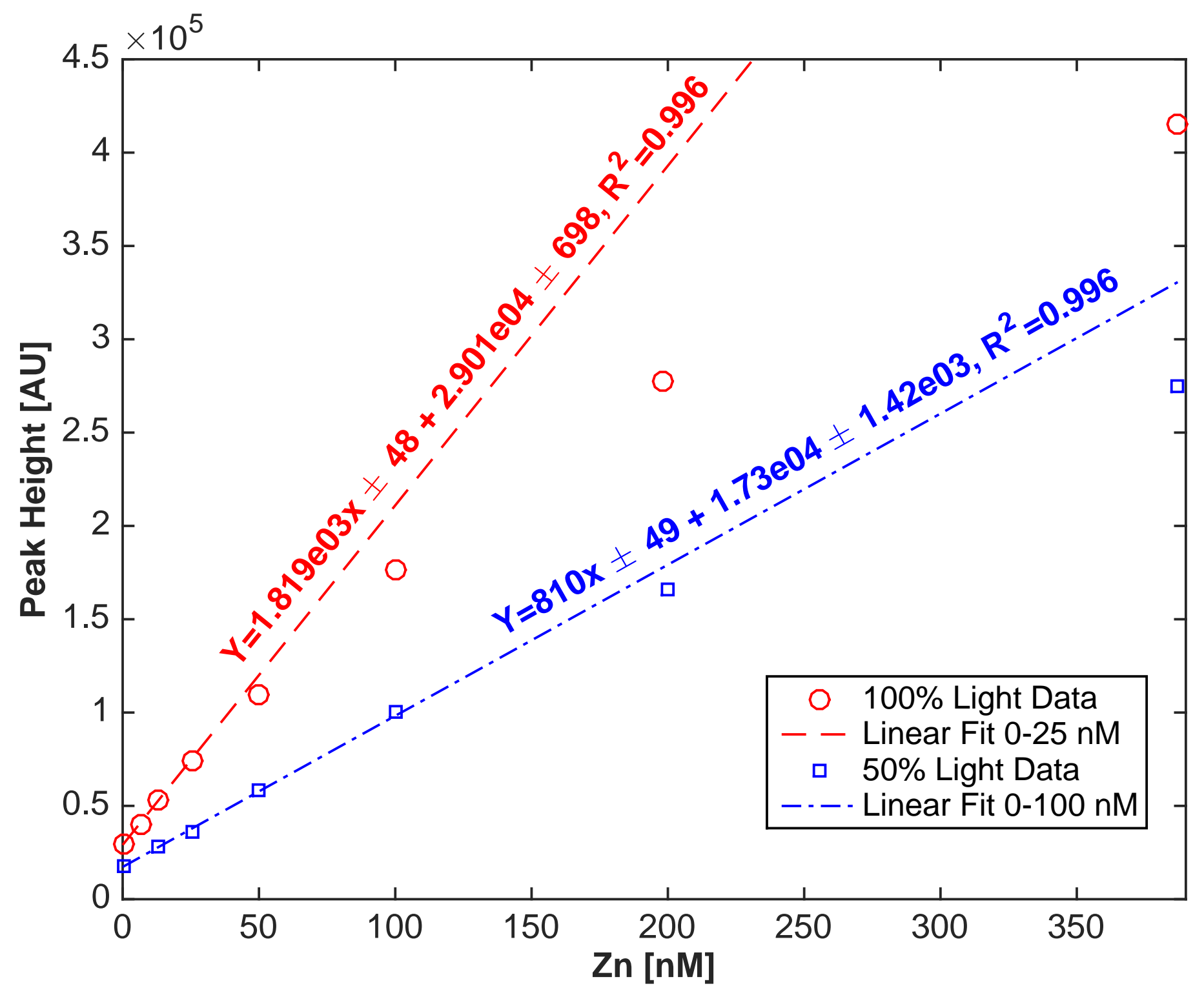




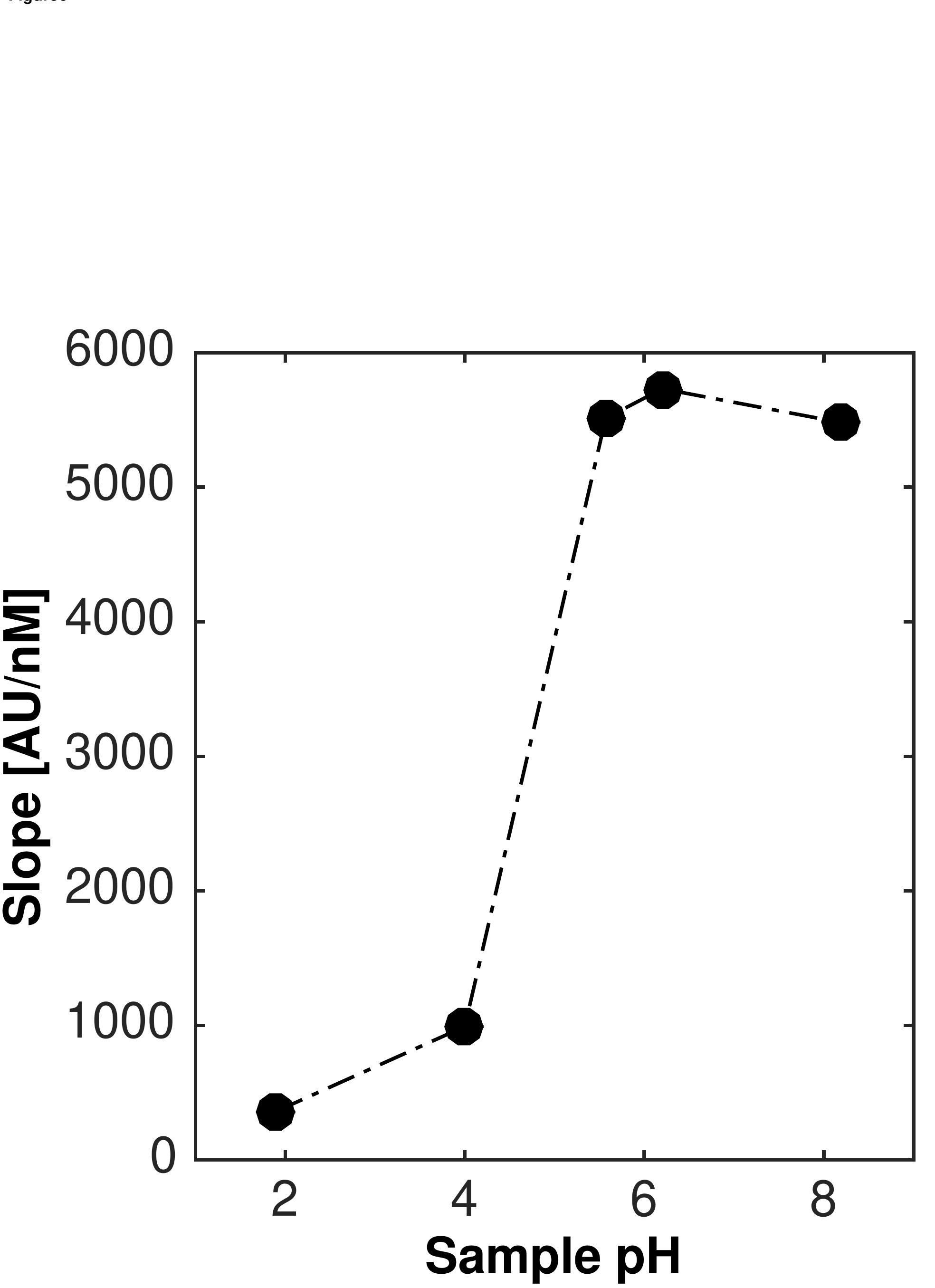

Figure6

6000

5000

2000

ע

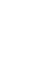

rest

4000

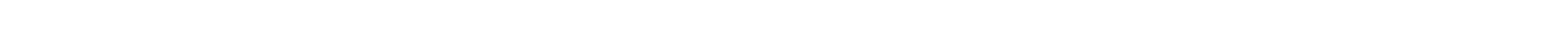




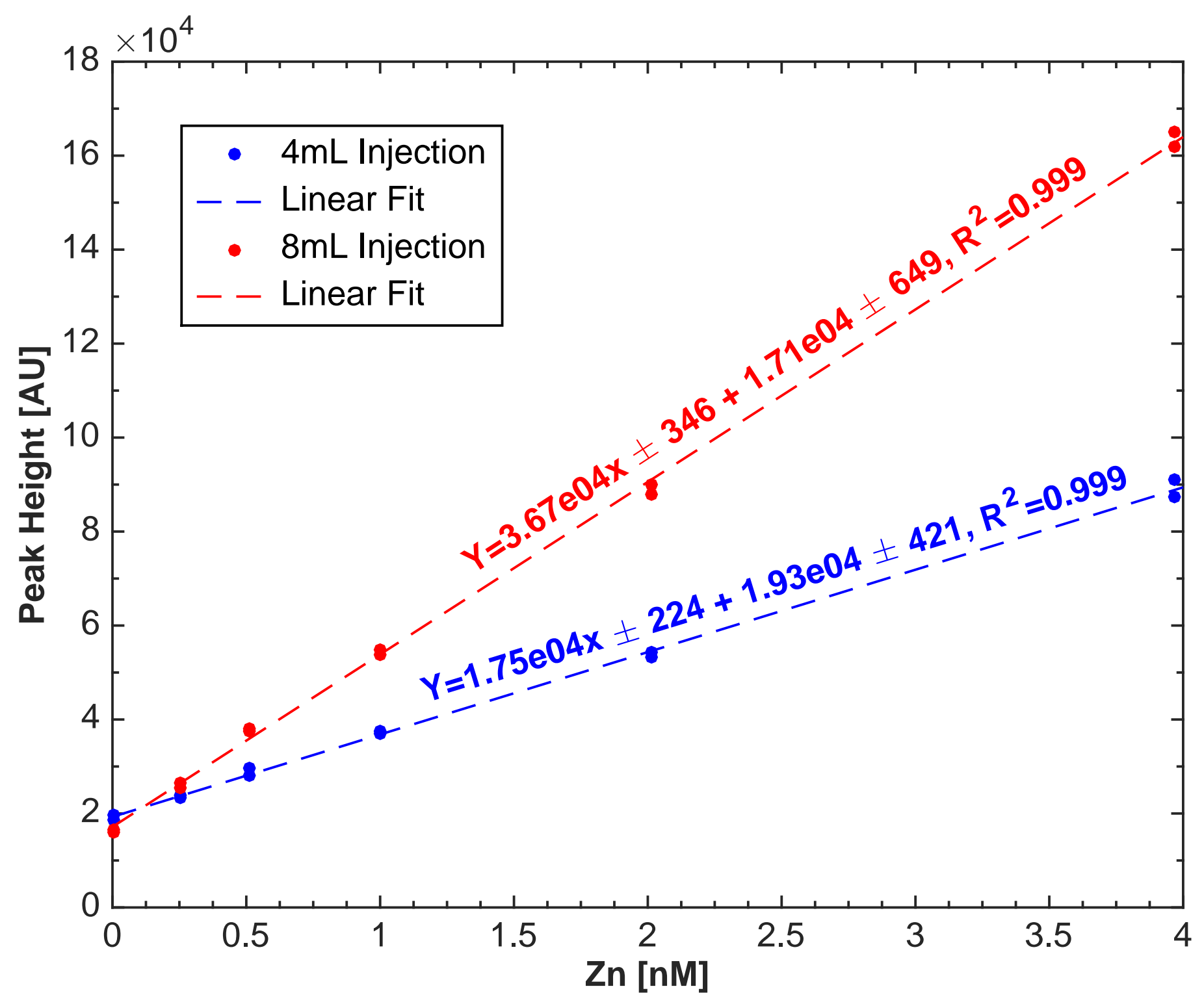




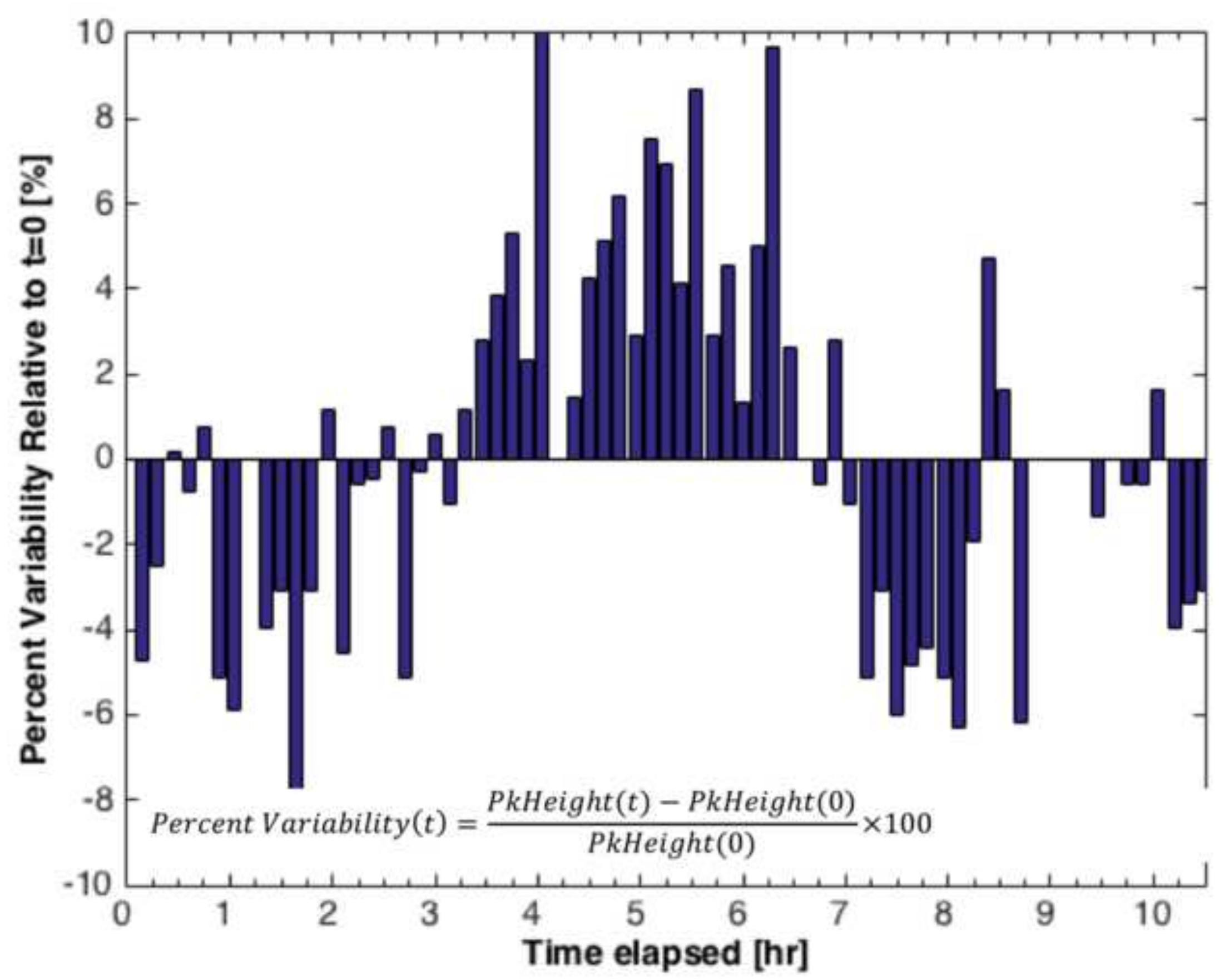

Time elapsed [hr] 
